\title{
Does the 'Higgs' have Spin Zero?
}

\author{
John Ellis ${ }^{a, b}$ and Dae Sung Hwang ${ }^{c}$ \\ ${ }^{a}$ Theoretical Particle Physics and Cosmology Group, Physics Department, \\ King's College London, Strand, London, U.K. \\ b Theory Division, Physics Department, CERN, \\ 1211 Geneva 23, Switzerland \\ ${ }^{c}$ Department of Physics, Sejong University, \\ Seoul 143-747, South Korea \\ E-mail: John.Ellis@cern.ch, dshwang@sejong.ac.kr
}

Abstract: The Higgs boson is predicted to have spin zero. The ATLAS and CMS experiments have recently reported of an excess of events with mass $\sim 125 \mathrm{GeV}$ that has some of the characteristics expected for a Higgs boson. We address the questions whether there is already any evidence that this excess has spin zero, and how this possibility could be confirmed in the near future. The excess observed in the $\gamma \gamma$ final state could not have spin one, leaving zero and two as open possibilities. We review the angular distribution of $\gamma \gamma$ pairs from the decays of a graviton-like spin-two boson produced in gluon-gluon collisions, which is well-defined and distinct from the spin-zero case. We also calculate the distributions for lepton pairs that would be produced in the $W W^{*}$ decays of a spin-two boson, which are very different from those in Higgs decays, and note that the kinematics of the event selection currently used in the analysis of the $W W^{*}$ final state have reduced efficiency for spin two.

Keywords: Higgs Physics, Beyond Standard Model, Standard Model

ARXIV EPRINT: 1202.6660 


\section{Contents}

1 Introduction 1

2 Preliminaries 3

2.1 Production kinematics 3

2.2 Polarization states 4

3 The process $g g \rightarrow X_{2} \rightarrow \gamma \gamma \quad 5$

3.1 Preliminaries 5

$\begin{array}{lll}3.2 & \text { Calculation of differential cross section } & 7\end{array}$

4 The process $g g \rightarrow X_{2} \rightarrow W^{-} W^{+} \rightarrow \ell^{-} \ell^{+} \bar{\nu} \nu \quad 10$

4.1 Lepton angular distributions in $W$ decays 10

$\begin{array}{lll}\text { 4.1.1 } W^{-} \rightarrow \ell^{-} \bar{\nu} & 10\end{array}$

$\begin{array}{lll}\text { 4.1.2 } W^{+} \rightarrow \ell^{+} \nu & 10\end{array}$

4.2 Angular distributions in $g g \rightarrow X_{2}\left(X_{0}\right) \rightarrow W^{-} W^{+} \rightarrow \ell^{-} \ell^{+} \bar{\nu} \nu \quad 12$

4.3 Angular correlations in $g g \rightarrow X_{2} \rightarrow W^{-} W^{+} \rightarrow \ell^{-} \ell^{+} \bar{\nu} \nu \quad 13$

$\begin{array}{lll}\text { 4.3.1 }\left|J J^{z}\right\rangle=|2+2\rangle & 13\end{array}$

4.3.2 $\left|J J^{z}\right\rangle=|2-2\rangle \quad 13$

4.4 Angular correlations in $g g \rightarrow X_{0} \rightarrow W^{-} W^{+} \rightarrow \ell^{-} \ell^{+} \bar{\nu} \nu \quad 16$

$\begin{array}{lll}\text { 4.5 Dilepton invariant mass distributions } & 17\end{array}$

$\begin{array}{lll}5 & \text { Summary } & 17\end{array}$

\section{Introduction}

The Higgs boson is predicted to have spin zero. Since all known elementary particles have non-zero spin, this is a crucial property to be checked by experiment before one could claim that the quest for this 'Holy Grail' of particle physics has been concluded successfully. Reflecting the importance of this issue, there have been many studies of the potential of the LHC experiments for measuring the spin of any candidate for the Higgs boson [1-14].

Most of these papers proposed to look at spin correlations in $Z Z$ or $Z Z^{*}$ decays using four-charged-lepton final states $[2,4,5,7,9-14]$. To our knowledge, the only published study of a spin-two state $X$ decaying into $\gamma \gamma$ has been [11], see appendix A. Ref. [6] studied the production of $X \rightarrow W^{+} W^{-} \rightarrow \ell^{+} \ell^{-} \nu \bar{\nu}$ final states via vector-boson fusion, distributions for the transverse angles of charged leptons in particles $X \rightarrow W^{+} W^{-} \rightarrow$ $\ell^{+} \ell^{-} \nu \bar{\nu}$ decays were considered in [7], and high-mass $X \rightarrow W^{+} W^{-} \rightarrow \ell^{+} \ell^{-} \nu \bar{\nu}$ decays were considered in [14]. However, in all these papers only the cases where $X$ has spin 0 or 1 were considered. Ref. [11] discussed spin-2 decays into $W^{+} W^{-}$but did not discus in detail 
charged-lepton angular distributions. Refs. $[1,8]$ considered production in $e^{+} e^{-}$collisions, in association with $Z$ and $\bar{t} t$, respectively.

The ATLAS [15-17] and CMS [18-25] collaborations have recently reported evidence for excesses in $\gamma \gamma$ and $Z Z^{*}$ that are consistent with expectations for a Standard Model Higgs boson, an interpretation supported by broader enhancements of less significance in $W W^{*}, \tau \tau$ and $\bar{b} b$ final states. The statistics in $Z Z^{*}$ decays are as yet insufficient for an attempt to constrain the 'Higgs' spin, so in this paper we consider other ways to obtain an indication what it may be.

A spin-one state cannot decay into two identical vector bosons, so a peak observed in the $\gamma \gamma$ final state must have spin zero or two. ${ }^{1}$ Fermion-antifermion final states could come from spin zero or spin one, ${ }^{2}$ so observation of a 'Higgs' signal in either of the $\tau \tau$ or $\bar{b} b$ final states would favour the spin-zero hypothesis over the spin-two option. However, so far only CMS reports any enhancements in these channels, and they are each $\leq 1 \sigma$ for a mass of $125 \mathrm{GeV}$ [18-25], so not conclusive at the present time. Accordingly, we consider here the $\gamma \gamma$ and $W W^{*} \rightarrow \ell^{+} \ell^{-} \nu \bar{\nu}$ final states, which have been observed with greater significance by both ATLAS and CMS.

Under the assumption that $\mathrm{P}$-wave fermion-antifermion collisions can be neglected, ${ }^{3}$ a spin-two particle could be produced either by gluon-gluon collisions or by vector-boson fusion. We consider here the production of a hypothetical spin-two particle $X_{2}$ via gluongluon fusion, which is the dominant production mechanism for producing a Higgs boson weighing $\sim 125 \mathrm{GeV}$. For definiteness, we assume that the $X_{2}$ couplings are of the same form as a massive Kaluza-Klein graviton [27-29] and in a string model [26], though without committing ourselves to either framework. ${ }^{4}$

We first review the angular distribution for $g g \rightarrow X_{2} \rightarrow \gamma \gamma$ (see appendix A of [11]), recalling that if graviton-like couplings are assumed the final-state angular distribution in the $X_{2}$ centre of mass system is completely determined. It is suppressed at large angles relative to the beams, and hence is in principle distinct from the isotropic distribution predicted for spin-zero Higgs decay.

We then turn to the $X_{2} \rightarrow W W^{*} \rightarrow \ell^{+} \ell^{-} \nu \bar{\nu}$ final state, again assuming production by gluon-gluon fusion and the same couplings as in massive graviton models [27-29]. We note that the ATLAS and CMS searches for $W W^{*} \rightarrow \ell^{+} \ell^{-} \nu \bar{\nu}$ final states [15-25] already incorporate a hypothesis about the spin of the 'Higgs' candidate. They make use of the observation in [31] that a spin-zero particle decaying into $W W$ (or $W W^{*}$ ) would yield final states in which the $W^{+}$and $W^{-}$would have opposite polarizations. Since the $W^{-}$decays exclusively into left-handed leptons, whereas the $W^{+}$decays exclusively into right-handed leptons, the anticorrelation between the $W^{ \pm}$polarizations expected in spin-zero Higgs decay would be transferred into a correlation between the momenta of the charged leptons

\footnotetext{
${ }^{1}$ In principle, one could consider also higher spins, but these would entail production and decay mechanisms involving orbital angular momentum factors that we ignore here.

${ }^{2}$ Again neglecting orbital angular momentum.

${ }^{3}$ This is not necessarily the case in string models [26].

${ }^{4}$ It was shown in [30] that this form is unique if the $X_{2}$ couplings to pairs of vector bosons are of dimension five (the lowest possible) and one assumes gauge and CP invariance.
} 
in their decays. This correlation would manifest itself in the distributions of relative $\ell^{ \pm}$ polar angles and a preference for a small azimuthal angle between the $\ell^{+} \ell^{-}$pair, $\phi_{\ell^{+} \ell^{-}}$, with a relatively small invariant mass, $m_{\ell^{+} \ell^{-}}$. Both ATLAS and CMS select events with cuts based on these observations [15-25].

We study the types of $\ell^{+} \ell^{-}$correlations to be expected in the $W W^{*} \rightarrow \ell^{+} \ell^{-} \nu \bar{\nu}$ decays of a spin-two state. We find that their momenta tend to be anticorrelated, with distinctive features in both polar and azimuthal angle distributions, and hence quite distinct from those expected for the decays of a spin-zero state. Hence, the observation (or not) of Higgs-like $\ell^{+} \ell^{-}$correlations in $W W^{*}$ final states could help provide evidence that ATLAS and CMS may be observing a spin-zero (-two) state. This possibility should be pursued with experimental simulations of spin-two $W W^{*} \rightarrow \ell^{+} \ell^{-} \nu \bar{\nu}$ decays using the results presented here, which would indicate how much data would be needed to confirm the result with a significant degree of confidence.

\section{Preliminaries}

\subsection{Production kinematics}

Ideally, one would prefer to perform such a 'Higgs' spin analysis in the most modelindependent way possible. However, the density matrix of a massive spin-two particle has many parameters, and the available statistics limit the complexity of the hypotheses one can test currently, so we are led to make motivated simplifying assumptions about the possible production mechanism of a massive spin-two state. Bosons are generally produced in $p p$ collisions by $\bar{q} q, g g$ or $W W / Z Z$ collisions. However, neglecting orbital angular momentum, $\bar{q} q$ collisions can produce only spin-zero or -one states, so we are left with $g g$ and $W W / Z Z$ collisions. Since $g g$ collisions are much more copious and simpler to analyze, we focus on them.

Neglecting initial-state transverse momentum and radiation, we may regard the gluons as massless spin-one particles whose momenta are aligned with the collision axis. As such, if one quantizes angular momentum along this axis, they are equally likely to be in the helicity states $|1, \pm 1\rangle$. We assume that there is no coherence between the final states in which different gluon helicity states collide. Therefore the initial-state combinations $|1,+1\rangle|1,+1\rangle,|1,+1\rangle|1,-1\rangle,|1,-1\rangle|1,+1\rangle$ and $|1,-1\rangle|1,-1\rangle$ are equally likely. Accordingly, the $g g$ initial states are a combination of the $|2,+2\rangle,|2,-2\rangle$ and $|2,0\rangle$ polarization states, described by a spin-two density matrix $\rho_{2}$ that has only diagonal entries with relative weights

$$
\rho_{i}=\frac{3}{7}\left(|22\rangle\left\langle 22\left|+\frac{1}{3}\right| 20\right\rangle\langle 20|+| 2-2\rangle\langle 2-2|\right),
$$

where the relative normalization of the $J_{z}=0$ component is determined by the ClebschGordan coefficients $\langle 2,0|| 1, \pm 1\rangle|1, \mp 1\rangle=1 / \sqrt{6}$.

We explore in the following sections the consequences of this observation for the possible decays of a hypothetical spin-two particle $X_{2}$ into $\gamma \gamma$ and $W^{+} W^{-}$final states at the LHC. The $|20\rangle\langle 20|$ component in the density matrix does not contribute if graviton-like couplings are assumed [26], as done here. 


\subsection{Polarization states}

Before discussing further the kinematics and dynamics of $X_{2}$ production and decay, we briefly review and establish our notation for the polarization states of the spin-one and -two particles appearing in our analysis.

A massive spin-one particle with momentum $p^{\mu}=\left(p^{0}, p^{1}, p^{2}, p^{3}\right)=(E, 0,0, p)$ has three independent polarization states given by

$$
\begin{aligned}
\epsilon^{+\mu} & =\left(0,-\frac{1}{\sqrt{2}},-\frac{i}{\sqrt{2}}, 0\right), \\
\epsilon^{-\mu} & =\left(0,+\frac{1}{\sqrt{2}},-\frac{i}{\sqrt{2}}, 0\right), \\
\epsilon^{0 \mu} & =\left(\frac{p}{m}, 0,0, \frac{E}{m}\right) .
\end{aligned}
$$

If we work in the Lorentz frame where the vector particle is at rest, so that $p^{\mu}=$ $\left(p^{0}, p^{1}, p^{2}, p^{3}\right)=(m, 0,0,0)$, the three polarization vectors are given by $(2.2),(2.3)$, and for $(2.4)$

$$
\epsilon^{0 \mu}=(0,0,0,1)
$$

The polarization vectors $\epsilon^{+\mu}, \epsilon^{-\mu}$ and $\epsilon^{0 \mu}$ correspond to the quantum states $|1,+1\rangle,|1,-1\rangle$ and $|1,0\rangle$, respectively, with the $z$-axis as the quantization axis.

We now consider the spin states of a spin-two particle $X_{2}$ with mass $m$, in its rest frame. The polarizations of $X_{2}$ can be represented by the following polarization tensors:

$$
\begin{aligned}
\epsilon^{s \mu \nu}= & (|2+2\rangle,|2,+1\rangle,|2,0\rangle,|2,-1\rangle,|2,-2\rangle) \\
= & \left(\epsilon^{+2 \mu \nu}, \epsilon^{+1 \mu \nu}, \epsilon^{0 \mu \nu}, \epsilon^{-1 \mu \nu}, \epsilon^{-2 \mu \nu}\right) \\
= & \left(\epsilon^{+\mu} \epsilon^{+\nu}, \frac{1}{\sqrt{2}}\left(\epsilon^{+\mu} \epsilon^{0 \nu}+\epsilon^{0 \mu} \epsilon^{+\nu}\right),\right. \\
& \left.\frac{1}{\sqrt{6}}\left(\epsilon^{+\mu} \epsilon^{-\nu}+\epsilon^{-\mu} \epsilon^{+\nu}+2 \epsilon^{0 \mu} \epsilon^{0 \nu}\right), \frac{1}{\sqrt{2}}\left(\epsilon^{-\mu} \epsilon^{0 \nu}+\epsilon^{0 \mu} \epsilon^{-\nu}\right), \epsilon^{-\mu} \epsilon^{-\nu}\right),
\end{aligned}
$$

where $\epsilon^{+\mu}, \epsilon^{-\mu}$ are given in (2.2) and (2.3), and $\epsilon^{0 \mu}$ is given in (2.5), since we work in the $X_{2}$ rest frame.

The polarization tensors given in (2.6) satisfy the following relations:

$$
\left(\epsilon^{s}\right)_{\mu}^{\mu}=0, \quad p_{\mu} \epsilon^{s \mu \nu}=0, \quad \epsilon^{s \mu \nu} \epsilon_{\mu \nu}^{s^{\prime}{ }^{*}}=\delta^{s s^{\prime}},
$$

and

$$
\sum_{s=-2}^{+2} \epsilon_{\mu \nu}^{s} \epsilon_{\alpha \beta}^{s *}=B_{\mu \nu \alpha \beta}
$$

where

$$
\begin{aligned}
B_{\mu \nu \alpha \beta}= & \left(\eta_{\mu \alpha}-\frac{p_{\mu} p_{\alpha}}{m^{2}}\right)\left(\eta_{\nu \beta}-\frac{p_{\nu} p_{\beta}}{m^{2}}\right)+\left(\eta_{\mu \beta}-\frac{p_{\mu} p_{\beta}}{m^{2}}\right)\left(\eta_{\nu \alpha}-\frac{p_{\nu} p_{\alpha}}{m^{2}}\right) \\
& -\frac{2}{3}\left(\eta_{\mu \nu}-\frac{p_{\mu} p_{\nu}}{m^{2}}\right)\left(\eta_{\alpha \beta}-\frac{p_{\alpha} p_{\beta}}{m^{2}}\right) .
\end{aligned}
$$


We further note for reference that the propagator of the spin-two massive $X_{2}$ particle is given by [27-29]

$$
i \Delta_{\mu \nu \alpha \beta}=\frac{i B_{\mu \nu \alpha \beta}}{p^{2}-m^{2}+i \varepsilon},
$$

though the denominator of this formula is not used in this paper, since we consider only on-shell resonant production of $X_{2}$.

\section{$3 \quad$ The process $g g \rightarrow X_{2} \rightarrow \gamma \gamma$}

\subsection{Preliminaries}

The three-point vertex for $X_{2} \gamma \gamma$ or $g g$ is illustrated in figure 1(a), the process $g g \rightarrow$ $X_{2} \rightarrow \gamma \gamma$ is illustrated in figure 1(b), and our notation for the kinematics is illustrated in figure 1(c). For definiteness, we use the following Feynman riule for the $X_{2} \gamma \gamma$ vertex, which was derived in $[27,28]$ for the coupling of a massive Kaluza-Klein graviton:

$$
-\frac{i}{M}\left(W_{\mu \nu \alpha \beta}^{(\gamma)}+W_{\nu \mu \alpha \beta}^{(\gamma)}\right)
$$

where $M$ is a normalization factor and

$$
\begin{aligned}
W_{\mu \nu \alpha \beta}^{(\gamma)}= & \frac{1}{2} \eta_{\mu \nu}\left(-k_{1} \cdot k_{2} \eta_{\alpha \beta}+k_{1 \beta} k_{2 \alpha}\right) \\
& +k_{1} \cdot k_{2} \eta_{\mu \alpha} \eta_{\nu \beta} \\
& -\eta_{\mu \alpha} k_{1 \beta} k_{2 \nu}-\eta_{\mu \beta} k_{1 \nu} k_{2 \alpha} \\
& +\eta_{\alpha \beta} k_{1 \mu} k_{2 \nu} .
\end{aligned}
$$

The $X_{2} g g$ vertex is identical, apart from a trivial color factor $\delta^{a b}$.

We work in the $X_{2}$ rest frame, take the beam direction as the $z$-axis, and write the momenta of the initial-state gluons as

$$
k_{1}^{\mu}=\left(k_{1}^{0}, k_{1}^{1}, k_{1}^{2}, k_{1}^{3}\right)=(k, 0,0, k), \quad k_{2}^{\mu}=\left(k_{2}^{0}, k_{2}^{1}, k_{2}^{2}, k_{2}^{3}\right)=(k, 0,0,-k) .
$$

We recall that the polarization vectors of the massless initial-state gluons are given by $(2.2)$ and (2.3).

We denote the unit spatial vectors in the coordinate system where (3.6), (2.2) and (2.3) apply for the initial-state gluons by $\hat{x}, \hat{y}$ and $\hat{z}$. We denote the momenta of the final-state photons by $k_{1}^{\prime}$ and $k_{2}^{\prime}$, and take the three-momentum vector of $k_{1}^{\prime}$ to lie along the $\hat{z}^{\prime}$ direction, where $\hat{x}^{\prime}, \hat{y}^{\prime}$ and $\hat{z}^{\prime}$ are given by

$$
\hat{x}^{\prime}=\cos \theta \hat{x}-\sin \theta \hat{z}, \quad \hat{z}^{\prime}=\sin \theta \hat{x}+\cos \theta \hat{z}, \quad \hat{y}^{\prime}=\hat{y},
$$

i.e., $\hat{z}^{\prime}$ is given by rotating $\hat{z}$ toward $\hat{x}$ by the angle $\theta$, as shown in figure $1(\mathrm{c})$. The momenta $k_{1}^{\prime}$ and $k_{2}^{\prime}$, as well as the polarization vectors of the final-state photons in the coordinate system $\hat{x}^{\prime}, \hat{y}^{\prime}$ and $\hat{z}^{\prime}$, are given by expressions identical to those given in (2.2), (2.3) and (3.6) for the initial-state gluons in the coordinate system $\hat{x}, \hat{y}$ and $\hat{z}$. Then, in the coordinate system $\hat{x}, \hat{y}$ and $\hat{z}$, we have

$$
k_{1}^{\prime \mu}=k(1, \sin \theta, 0, \cos \theta), \quad k_{2}^{\prime \mu}=k(1,-\sin \theta, 0,-\cos \theta),
$$



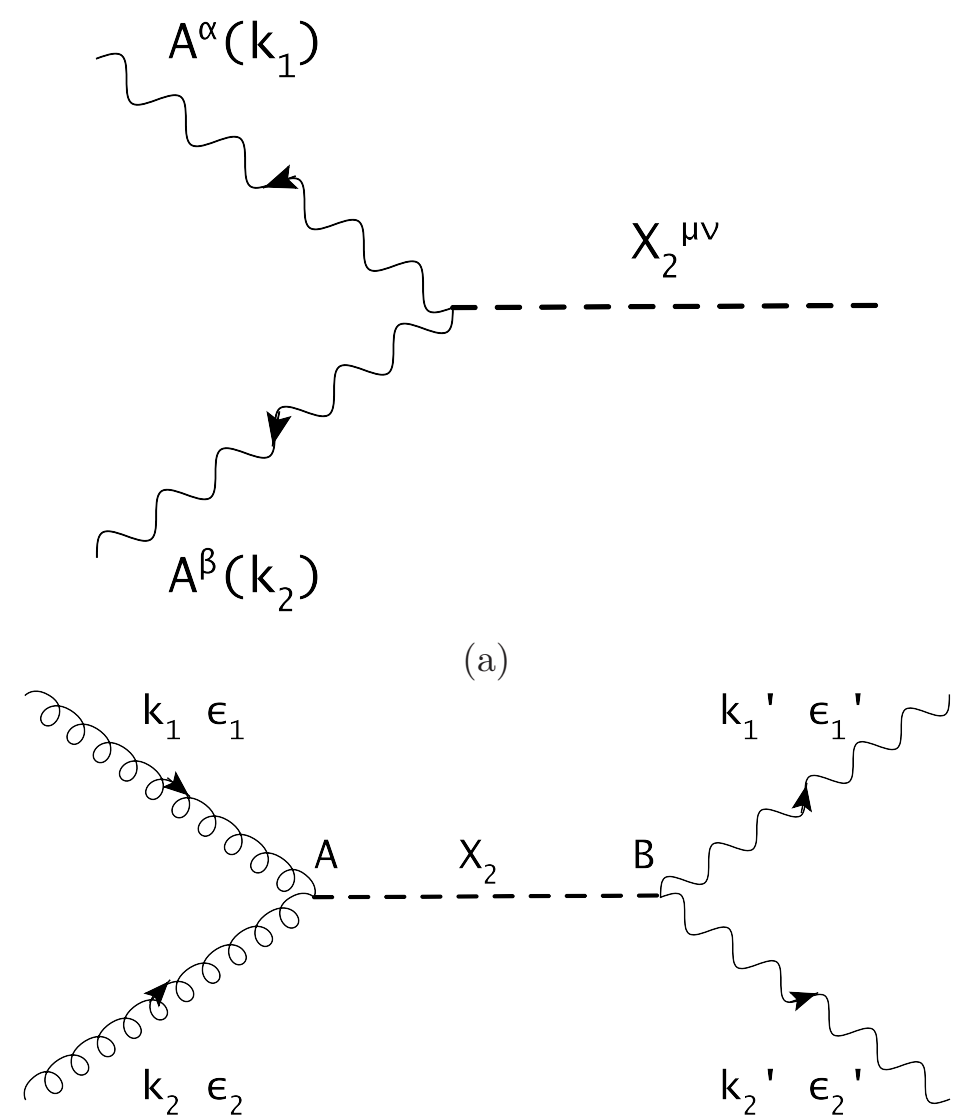

(b)

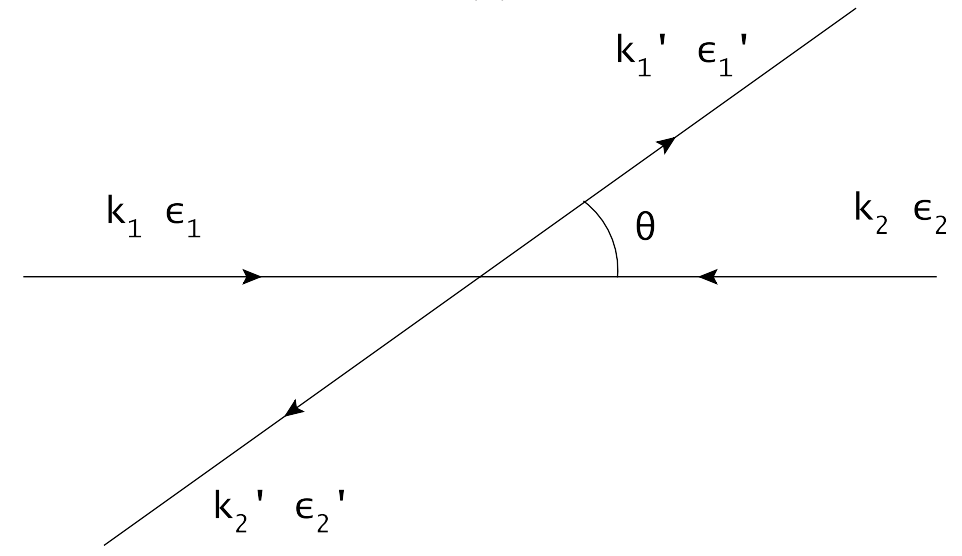

(c)

Figure 1. (a) The vertex coupling $X_{2}$ to two gauge fields, (b) Feynman diagram and (c) the kinematics for the process $g g \rightarrow X_{2} \rightarrow \gamma \gamma$.

and

$$
\epsilon^{\prime+\mu}=\left(0,-\frac{1}{\sqrt{2}} \cos \theta,-\frac{i}{\sqrt{2}}, \frac{1}{\sqrt{2}} \sin \theta\right), \quad \epsilon^{\prime-\mu}=\left(0, \frac{1}{\sqrt{2}} \cos \theta,-\frac{i}{\sqrt{2}},-\frac{1}{\sqrt{2}} \sin \theta\right) .
$$


The three rotated polarizations of $X_{2}$ are represented by the two given in (3.9) and by

$$
\epsilon^{\prime 0 \mu}=(0, \sin \theta, 0, \cos \theta)
$$

We note that the polarization vectors $\epsilon^{\prime \mu}$ are labeled by \pm , according to the component of the photon spin along the $k_{1}^{\prime}$ direction, just as the $\epsilon^{\mu}$ are labeled by the component of the gluon spin along the $k_{1}$ direction. The amplitude of the process $g g \rightarrow X_{2} \rightarrow \gamma \gamma$ is of the form

$$
A\left(\epsilon_{1}^{\prime} \epsilon_{2}^{\prime} ; \epsilon_{1} \epsilon_{2}\right) \propto \epsilon_{1}^{\prime a *} \epsilon_{2}^{\prime b *} W_{a b \rho \sigma}\left(\sum_{s=-2}^{+2} \epsilon^{s \rho \sigma} \epsilon^{s \mu \nu *}\right) W_{\mu \nu \alpha \beta} \epsilon_{1}^{\alpha} \epsilon_{2}^{\beta},
$$

where the vertex $W_{\mu \nu \alpha \beta}$ is given in (3.1).

\subsection{Calculation of differential cross section}

We calculate the amplitude for $g g \rightarrow X_{2} \rightarrow \gamma \gamma$ when the initial gluon polarization state is one of $\epsilon_{1}^{+} \epsilon_{2}^{+}, \epsilon_{1}^{-} \epsilon_{2}^{-}, \epsilon_{1}^{+} \epsilon_{2}^{-}$and $\epsilon_{1}^{-} \epsilon_{2}^{+}$, and the final photon polarization state is one of $\epsilon_{1}^{\prime+} \epsilon_{2}^{\prime+}$, $\epsilon_{1}^{\prime-} \epsilon_{2}^{\prime-}, \epsilon_{1}^{\prime+} \epsilon_{2}^{\prime-}$ and $\epsilon_{1}^{\prime-} \epsilon_{2}^{\prime+}$, via the Feynman diagram drawn in figure 1(b).

Using the the first equation in (2.7), we see that (3.2) does not contribute to the amplitudes. Moreover, we see from (2.2), (2.3) and (3.6), that $k_{1} \cdot \epsilon_{2}=k_{2} \cdot \epsilon_{1}=0$ and hence (3.4) also does not contribute to the amplitudes. Therefore, only the terms (3.3) and (3.5) in $W_{\mu \nu \alpha \beta}^{(\gamma)}$ may contribute to the amplitudes.

We find that the amplitude for $g g \rightarrow X_{2} \rightarrow \gamma \gamma$ is non-zero only when both of the following two conditions are satisfied: (1) the initial gluon polarization state is one of $\epsilon_{1}^{+} \epsilon_{2}^{+}$ and $\epsilon_{1}^{-} \epsilon_{2}^{-}$, and (2) the final photon polarization state is one of $\epsilon_{1}^{\prime+} \epsilon_{2}^{\prime+}$ and $\epsilon_{1}^{\prime-} \epsilon_{2}^{\prime-}$. On the other hand, the amplitude is zero either when the initial gluon polarization state is one of $\epsilon_{1}^{+} \epsilon_{2}^{-}$and $\epsilon_{1}^{-} \epsilon_{2}^{+}$, or when the final photon polarization state is one of $\epsilon_{1}^{\prime+} \epsilon_{2}^{\prime-}$ and $\epsilon_{1}^{\prime-} \epsilon_{2}^{\prime+}$. Thus the only possible initial and final helicity states are $|22\rangle$ and $|2-2\rangle$, with no contribution from $|20\rangle$.

We consider the vertex $g g \rightarrow X_{2}$ in the process $g g \rightarrow X_{2} \rightarrow \gamma \gamma$, which corresponds to the vertex $A$ in figure 1(b). When the expression (3.5), i.e., $\eta_{\alpha \beta} k_{1 \mu} k_{2 \nu}$, is attached at this vertex, this vertex is non-zero only when the initial gluon polarization state is $\epsilon_{1}^{+} \epsilon_{2}^{-}$or $\epsilon_{1}^{-} \epsilon_{2}^{+}$, since $\eta_{\alpha \beta} \epsilon_{1}^{+\alpha} \epsilon_{2}^{-\beta}=\eta_{\alpha \beta} \epsilon_{1}^{-\alpha} \epsilon_{2}^{+\beta}=1$ and $\eta_{\alpha \beta} \epsilon_{1}^{+\alpha} \epsilon_{2}^{+\beta}=\eta_{\alpha \beta} \epsilon_{1}^{-\alpha} \epsilon_{2}^{-\beta}=0$. Then, using $\epsilon^{0 \mu \nu *} k_{1 \mu} k_{2 \nu}=\frac{2}{\sqrt{6}}\left(\epsilon^{0 *} \cdot k_{1}\right)\left(\epsilon^{0 *} \cdot k_{2}\right)=\frac{2}{\sqrt{6}}(-k)(+k)=-\frac{2}{\sqrt{6}} k^{2}$ and $\epsilon^{(s \neq 0) \mu \nu *} k_{1 \mu} k_{2 \nu}=0$, the amplitude (3.11) for this vertex becomes

$$
\begin{aligned}
& \epsilon_{1}^{\prime a *} \epsilon_{2}^{\prime b *} W_{a b \rho \sigma}\left(\sum_{s=-2}^{+2} \epsilon^{s \rho \sigma} \epsilon^{s \mu \nu *}\right)\left(\eta_{\alpha \beta} k_{1 \mu} k_{2 \nu}\right) \epsilon_{1}^{+\alpha} \epsilon_{2}^{-\beta} \\
& =\epsilon_{1}^{\prime a *} \epsilon_{2}^{\prime b *} W_{a b \rho \sigma}\left(\sum_{s=-2}^{+2} \epsilon^{s \rho \sigma} \epsilon^{s \mu \nu *}\right)\left(\eta_{\alpha \beta} k_{1 \mu} k_{2 \nu}\right) \epsilon_{1}^{-\alpha} \epsilon_{2}^{+\beta} \\
& =\epsilon_{1}^{a *} \epsilon_{2}^{\prime b *} W_{a b \rho \sigma} \epsilon^{0 \rho \sigma}\left(-\frac{2}{\sqrt{6}} k^{2}\right),
\end{aligned}
$$


and

$$
\begin{aligned}
& \epsilon_{1}^{\prime a *} \epsilon_{2}^{\prime b *} W_{a b \rho \sigma}\left(\sum_{s=-2}^{+2} \epsilon^{s \rho \sigma} \epsilon^{s \mu \nu *}\right)\left(\eta_{\alpha \beta} k_{1 \mu} k_{2 \nu}\right) \epsilon_{1}^{+\alpha} \epsilon_{2}^{+\beta} \\
& =\epsilon_{1}^{\prime a *} \epsilon_{2}^{\prime b *} W_{a b \rho \sigma}\left(\sum_{s=-2}^{+2} \epsilon^{s \rho \sigma} \epsilon^{s \mu \nu *}\right)\left(\eta_{\alpha \beta} k_{1 \mu} k_{2 \nu}\right) \epsilon_{1}^{-\alpha} \epsilon_{2}^{-\beta} \\
& =0 .
\end{aligned}
$$

When the expression (3.3), i.e., $k_{1} \cdot k_{2} \eta_{\mu \alpha} \eta_{\nu \beta}$, is attached at this vertex, using $\epsilon^{+*} \cdot \epsilon_{1}^{+}=$ $\epsilon^{-*} \cdot \epsilon_{1}^{-}=\epsilon^{+*} \cdot \epsilon_{2}^{+}=\epsilon^{-*} \cdot \epsilon_{2}^{-}=-1$ and $k_{1} \cdot k_{2}=2 k^{2}$, we find

$$
\begin{aligned}
& \epsilon_{1}^{\prime a *} \epsilon_{2}^{\prime b *} W_{a b \rho \sigma}\left(\sum_{s=-2}^{+2} \epsilon^{s \rho \sigma} \epsilon^{s \mu \nu *}\right)\left(k_{1} \cdot k_{2} \eta_{\mu \alpha} \eta_{\nu \beta}\right) \epsilon_{1}^{+\alpha} \epsilon_{2}^{-\beta} \\
& =\epsilon_{1}^{\prime a *} \epsilon_{2}^{\prime b *} W_{a b \rho \sigma}\left(\sum_{s=-2}^{+2} \epsilon^{s \rho \sigma} \epsilon^{s \mu \nu *}\right)\left(k_{1} \cdot k_{2} \eta_{\mu \alpha} \eta_{\nu \beta}\right) \epsilon_{1}^{-\alpha} \epsilon_{2}^{+\beta} \\
& =\epsilon_{1}^{\prime a *} \epsilon_{2}^{\prime b *} W_{a b \rho \sigma} \epsilon^{0 \rho \sigma}\left(+\frac{1}{\sqrt{6}} 2 k^{2}\right)
\end{aligned}
$$

and

$$
\begin{aligned}
& \epsilon_{1}^{\prime a *} \epsilon_{2}^{\prime b *} W_{a b \rho \sigma}\left(\sum_{s=-2}^{+2} \epsilon^{s \rho \sigma} \epsilon^{s \mu \nu *}\right)\left(k_{1} \cdot k_{2} \eta_{\mu \alpha} \eta_{\nu \beta}\right) \epsilon_{1}^{+\alpha} \epsilon_{2}^{+\beta}=\epsilon_{1}^{\prime a *} \epsilon_{2}^{\prime b *} W_{a b \rho \sigma} \epsilon^{+2 \rho \sigma}\left(2 k^{2}\right) \\
& \epsilon_{1}^{\prime a *} \epsilon_{2}^{\prime b *} W_{a b \rho \sigma}\left(\sum_{s=-2}^{+2} \epsilon^{s \rho \sigma} \epsilon^{s \mu \nu *}\right)\left(k_{1} \cdot k_{2} \eta_{\mu \alpha} \eta_{\nu \beta}\right) \epsilon_{1}^{-\alpha} \epsilon_{2}^{-\beta}=\epsilon_{1}^{\prime a *} \epsilon_{2}^{\prime b *} W_{a b \rho \sigma} \epsilon^{-2 \rho \sigma}\left(2 k^{2}\right) .
\end{aligned}
$$

Combining (3.12) to (3.15), we have

$$
\begin{aligned}
& \epsilon_{1}^{\prime a *} \epsilon_{2}^{\prime b *} W_{a b \rho \sigma}\left(\sum_{s=-2}^{+2} \epsilon^{s \rho \sigma} \epsilon^{s \mu \nu *}\right)\left(k_{1} \cdot k_{2} \eta_{\mu \alpha} \eta_{\nu \beta}+\eta_{\alpha \beta} k_{1 \mu} k_{2 \nu}\right) \epsilon_{1}^{+\alpha} \epsilon_{2}^{-\beta} \\
& =\epsilon_{1}^{a *} \epsilon_{2}^{\prime b *} W_{a b \rho \sigma}\left(\sum_{s=-2}^{+2} \epsilon^{s \rho \sigma} \epsilon^{s \mu \nu *}\right)\left(k_{1} \cdot k_{2} \eta_{\mu \alpha} \eta_{\nu \beta}+\eta_{\alpha \beta} k_{1 \mu} k_{2 \nu}\right) \epsilon_{1}^{-\alpha} \epsilon_{2}^{+\beta} \\
& =0
\end{aligned}
$$

and

$$
\begin{gathered}
\epsilon_{1}^{\prime a *} \epsilon_{2}^{\prime b *} W_{a b \rho \sigma}\left(\sum_{s=-2}^{+2} \epsilon^{s \rho \sigma} \epsilon^{s \mu \nu *}\right)\left(k_{1} \cdot k_{2} \eta_{\mu \alpha} \eta_{\nu \beta}+\eta_{\alpha \beta} k_{1 \mu} k_{2 \nu}\right) \epsilon_{1}^{+\alpha} \epsilon_{2}^{+\beta} \\
=\epsilon_{1}^{\prime a *} \epsilon_{2}^{\prime b *} W_{a b \rho \sigma} \epsilon^{+2 \rho \sigma}\left(2 k^{2}\right), \\
\epsilon_{1}^{\prime a *} \epsilon_{2}^{\prime b *} W_{a b \rho \sigma}\left(\sum_{s=-2}^{+2} \epsilon^{s \rho \sigma} \epsilon^{s \mu \nu *}\right)\left(k_{1} \cdot k_{2} \eta_{\mu \alpha} \eta_{\nu \beta}+\eta_{\alpha \beta} k_{1 \mu} k_{2 \nu}\right) \epsilon_{1}^{-\alpha} \epsilon_{2}^{-\beta} \\
=\epsilon_{1}^{\prime a *} \epsilon_{2}^{\prime b *} W_{a b \rho \sigma} \epsilon^{-2 \rho \sigma}\left(2 k^{2}\right) .
\end{gathered}
$$


Equivalent results are obtained when the roles of the initial gluon polarization states and the final photon polarization states are exchanged in (3.16) and (3.17).

This analysis justifies the statement made at the beginning of this subsection, namely that the amplitude for $g g \rightarrow X_{2} \rightarrow \gamma \gamma$ is non-zero only when both of the following two conditions are satisfied: (1) the initial gluon polarization state is one of $\epsilon_{1}^{+} \epsilon_{2}^{+}$and $\epsilon_{1}^{-} \epsilon_{2}^{-}$, and (2) the final photon polarization state is one of $\epsilon_{1}^{\prime+} \epsilon_{2}^{\prime+}$ and $\epsilon_{1}^{\prime-} \epsilon_{2}^{\prime-}$. That is, the amplitude is zero either when the initial gluon polarization state is one of $\epsilon_{1}^{+} \epsilon_{2}^{-}$and $\epsilon_{1}^{-} \epsilon_{2}^{+}$, or when the final photon polarization state is one of $\epsilon_{1}^{\prime+} \epsilon_{2}^{\prime-}$ and $\epsilon_{1}^{\prime-} \epsilon_{2}^{\prime+}$.

We also see in (3.17) that in the non-zero amplitude found when both (3.3) and (3.5) are attached at both vertices in the Feynman diagram is the same as that obtained when only (3.3) is attached at both vertices in the Feynman diagram.

When the sum of (3.3) and (3.5), i.e., $\left(k_{1} \cdot k_{2} \eta_{\mu \alpha} \eta_{\nu \beta}+\eta_{\alpha \beta} k_{1 \mu} k_{2 \nu}\right)$, is attached at both vertices in the Feynman diagram for $g g \rightarrow X_{2} \rightarrow \gamma \gamma$ shown in figure 1(b), using (2.2), (2.3), (2.6) and (3.9) in (3.17), we find that the amplitudes (3.11) are given by $\left(4 k^{2} / M\right)^{2}$ times the following angular expressions:

$$
\begin{aligned}
& A\left(+^{\prime}+^{\prime} ;++\right)=A\left(-^{\prime}-^{\prime} ;--\right)=\frac{1}{4}(1+\cos \theta)^{2} \\
& A\left(-^{\prime}-^{\prime} ;++\right)=A\left(+^{\prime}+^{\prime} ;--\right)=\frac{1}{4}(1-\cos \theta)^{2} \\
& A\left(+^{\prime}-^{\prime} ;++\right)=A\left(-^{\prime}+^{\prime} ;++\right)=A\left(+^{\prime}-^{\prime} ;--\right)=A\left(-^{\prime}+^{\prime} ;--\right)=0 \\
& A\left(+^{\prime}+^{\prime} ;+-\right)=A\left(-^{\prime}-^{\prime} ;+-\right)=A\left(+^{\prime}+^{\prime} ;-+\right)=A\left(-^{\prime}-^{\prime} ;-+\right)=0 \\
& A\left(+^{\prime}-^{\prime} ;+-\right)=A\left(-^{\prime}+^{\prime} ;+-\right)=A\left(+^{\prime}-^{\prime} ;-+\right)=A\left(-^{\prime}+^{\prime} ;-+\right)=0 .
\end{aligned}
$$

The contributions of the two possible final polarization states $\epsilon_{1}^{\prime+} \epsilon_{2}^{\prime+}$ and $\epsilon_{1}^{\prime-} \epsilon_{2}^{\prime-}$ to the total $\gamma \gamma$ cross section $d \sigma / d \Omega$ are identical, and we have (as derived earlier in [11]):

$$
\frac{d \sigma}{d \Omega} \propto \frac{1}{4}+\frac{3}{2} \cos ^{2} \theta+\frac{1}{4} \cos ^{4} \theta
$$

which is plotted in figure 2 .

We see in figure 2 that the total $\gamma \gamma$ angular distribution in the $X_{2}$ centre-of-mass frame differs substantially from the isotropic angular distribution expected for the decay of a spin-zero particle such as the Higgs boson. In particular, the $\gamma \gamma$ final state is suppressed at large angles $\theta$ relative to the beams. This suggests that a careful study of the $\gamma \gamma$ angular distribution might offer some discrimination between the spin-two and spin-zero hypotheses. Any conclusion on this possibility would require a realistic simulation of the $\gamma \gamma$ signal in an LHC detector. However, we estimate that the centre-of-mass system of a photon pair can be reconstructed quite accurately, the dominant uncertainties probably being due to errors in the photon energy measurements, which are at the $1 \%$ level in both ATLAS and CMS. The preliminary results of simulation studies using Pythia and Delphes [34] support the expectation that the $\gamma \gamma$ angular distribution is little affected by detector effects. 


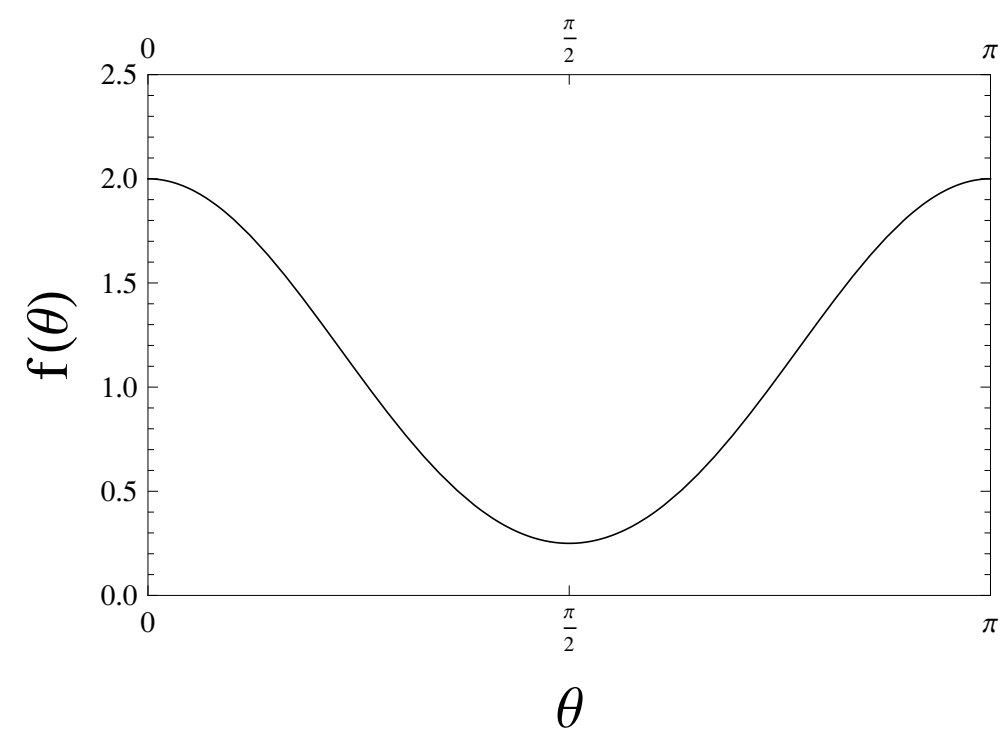

Figure 2. The $\gamma \gamma$ angular distribution of $d \sigma / d \Omega$ given in (3.23).

\section{The process $g g \rightarrow X_{2} \rightarrow W^{-} W^{+} \rightarrow \ell^{-} \ell^{+} \bar{\nu} \nu$}

\subsection{Lepton angular distributions in $W$ decays}

\subsection{1 $W^{-} \rightarrow \ell^{-} \bar{\nu}$}

As preparation for this section, we first consider the decay $W^{-} \rightarrow \ell^{-} \bar{\nu}$. We consider a $W^{-}$ at rest and denote the momenta of the final-state particles by

$$
\begin{aligned}
p_{\ell^{-}}^{\mu} & =\left(p, p \sin \theta_{1} \cos \phi_{1}, p \sin \theta_{1} \sin \phi_{1}, p \cos \theta_{1}\right), \\
p_{\bar{\nu}}^{\mu} & =\left(p,-p \sin \theta_{1} \cos \phi_{1},-p \sin \theta_{1} \sin \phi_{1},-p \cos \theta_{1}\right),
\end{aligned}
$$

where $p=|\vec{p}|$ and the $\ell^{-}$mass is ignored. The polarization vectors $\epsilon$ of $W^{-}$with the $z$-axis as the quantization axis are given by $(2.2),(2.3)$ and $\epsilon^{0 \mu}=(0,0,0,1)$. We calculate $\mathcal{M}_{1}=\bar{u}\left(p_{\ell^{-}}\right) \gamma^{\mu} \epsilon_{1 \mu}\left(1-\gamma_{5}\right) v\left(p_{\bar{\nu}}\right)$ and find the following results for $\mathcal{M}_{1} /(2 \sqrt{2} p)$ :

$$
\begin{array}{ll}
\text { for } \epsilon_{1}^{+}, & \left(1-\cos \theta_{1}\right) e^{+i \phi_{1}} \\
\text { for } \epsilon_{1}^{-}, & \left(1+\cos \theta_{1}\right) e^{-i \phi_{1}} \\
\text { for } \epsilon_{1}^{0}, & -\sqrt{2} \sin \theta_{1} .
\end{array}
$$

The differential cross section $d \sigma / d \Omega$ is proportional to $\left|\mathcal{M}_{1}\right|^{2}$ and the functions $f(\theta)=$ $\left|\mathcal{M}_{1} /(2 \sqrt{2} p)\right|^{2}$ for the three polarization states are plotted in figure 3 .

\subsection{2 $W^{+} \rightarrow \ell^{+} \nu$}

In the case of $W^{+}$at rest, we denote the momenta of the final-state particles by

$$
\begin{aligned}
p_{\ell^{+}}^{\mu} & =\left(p, p \sin \theta_{2} \cos \phi_{2}, p \sin \theta_{2} \sin \phi_{2}, p \cos \theta_{2}\right), \\
p_{\nu}^{\mu} & =\left(p,-p \sin \theta_{2} \cos \phi_{2},-p \sin \theta_{2} \sin \phi_{2},-p \cos \theta_{2}\right) .
\end{aligned}
$$




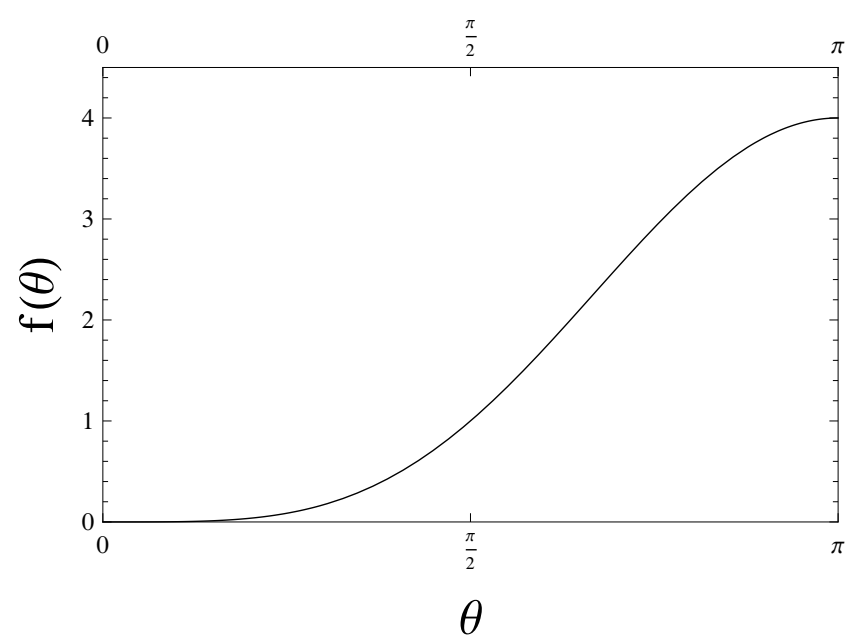

(a)

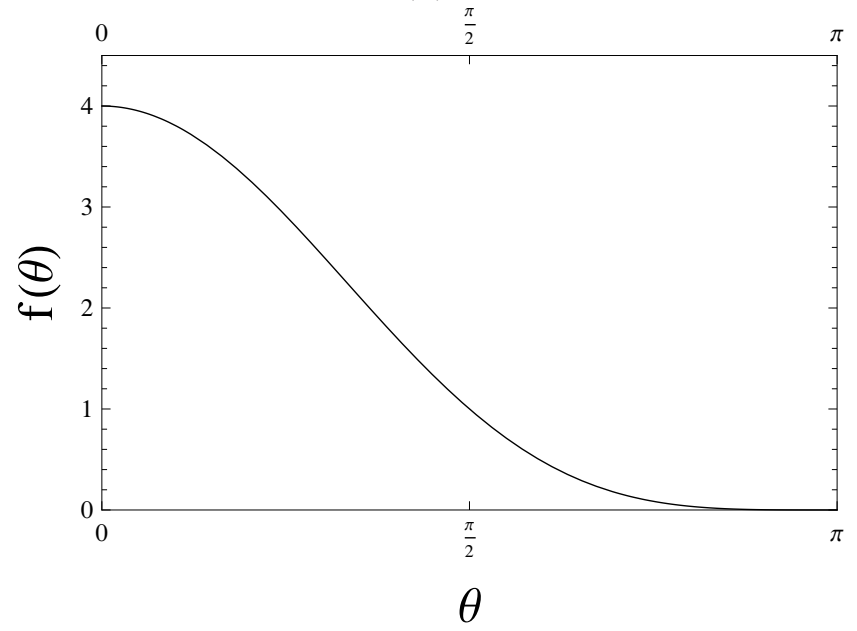

(b)

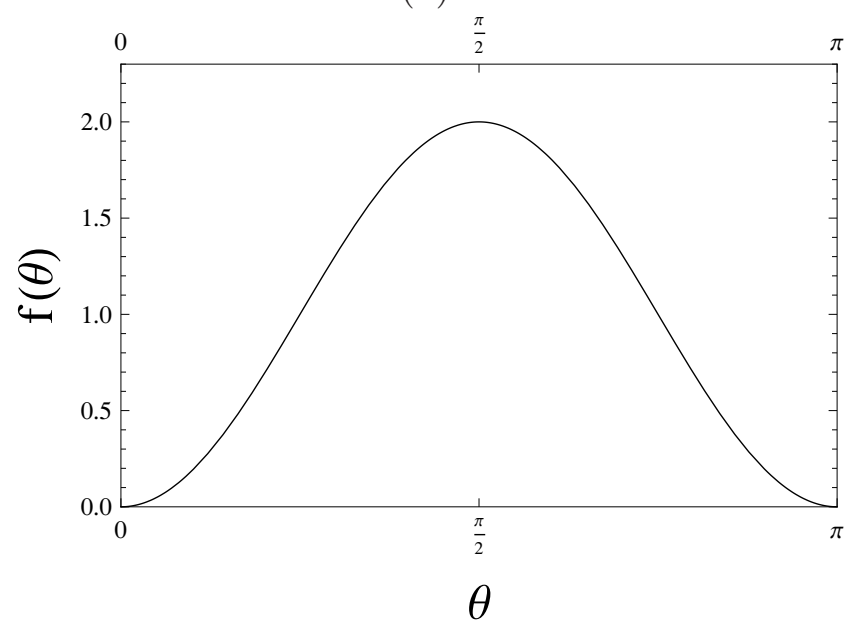

(c)

Figure 3. The decay angular distribution functions $f(\theta)$ in $W^{-} \rightarrow \ell^{-} \bar{\nu}$ decays from the $W^{-}$ polarization states given by (a) $\epsilon^{+}$, (b) $\epsilon^{-}$, and (c) $\epsilon^{0}$. 
We calculate $\mathcal{M}_{2}=\bar{u}\left(p_{\nu}\right) \gamma^{\mu} \epsilon_{2 \mu}\left(1-\gamma_{5}\right) v\left(p_{\ell^{+}}\right)$and find the following results for $\mathcal{M}_{2} /(2 \sqrt{2} p)$ :

$$
\begin{array}{ll}
\text { for } \epsilon_{2}^{+}, & -\left(1+\cos \theta_{2}\right) e^{+i \phi_{2}} \\
\text { for } \epsilon_{2}^{-}, & -\left(1-\cos \theta_{2}\right) e^{-i \phi_{2}} \\
\text { for } \epsilon_{2}^{0}, & -\sqrt{2} \sin \theta_{2} .
\end{array}
$$

\subsection{Angular distributions in $g g \rightarrow X_{2}\left(X_{0}\right) \rightarrow W^{-} W^{+} \rightarrow \ell^{-} \ell^{+} \bar{\nu} \nu$}

For simplicity, we assume that the $W^{-}$and $W^{+}$are at rest, corresponding to the case that $m_{X}=2 m_{W}$. In practice, we are interested in the decay of the possible particle with mass $\sim 125 \mathrm{GeV}$ reported by ATLAS and CMS, which would decay into one on-shell $W$ and one off-shell $W^{*} \rightarrow \ell \nu$. The structure of the $W \ell \nu$ decay matrix element would be dominated by the $W^{*}$ pole, favouring $\ell \nu$ invariant masses close to $m_{X}-m_{W}$ and hence small momenta for the $W$ and $W^{*}$ in the centre-of-mass frame of the decaying $X$ particle. The crude approximation of neglecting these momenta may serve to indicate whether in principle there could be significant differences between the decay angular distributions in $X_{2}$ and $X_{0}$ decay that could be investigated in more detailed simulations.

With this assumption, we denote the polarization vector and momentum of the $W^{-}$ $\left(W^{+}\right)$by $\epsilon_{1}$ and $k_{1}\left(\epsilon_{2}\right.$ and $\left.k_{2}\right)$, respectively. The polarization vectors $\epsilon_{1}$ and $\epsilon_{2}$ with the $z$-axis as the quantization axis are given by $(2.2),(2.3)$ and $\epsilon^{0 \mu}=(0,0,0,1)$ as before, and the momenta $k_{1}$ and $k_{2}$ are given by

$$
k_{1}^{\mu}=k_{2}^{\mu}=\left(k_{1}^{0}, k_{1}^{1}, k_{1}^{2}, k_{1}^{3}\right)=\left(m_{W}, 0,0,0\right)=\left(\frac{m}{2}, 0,0,0\right) .
$$

For the Feynman rule of the three-point vertex $X_{2} W^{-} W^{+}$, we use the following vertex which is given in $[28]$ :

$$
-\frac{i}{\bar{M}_{P}}\left(W_{\mu \nu \alpha \beta}^{(W)}+W_{\nu \mu \alpha \beta}^{(W)}\right)
$$

where

$$
\begin{aligned}
W_{\mu \nu \alpha \beta}^{(W)}= & \frac{1}{2} \eta_{\mu \nu}\left(-\left(m_{W}^{2}+k_{1} \cdot k_{2}\right) \eta_{\alpha \beta}+k_{1 \beta} k_{2 \alpha}\right) \\
& +\left(m_{W}^{2}+k_{1} \cdot k_{2}\right) \eta_{\mu \alpha} \eta_{\nu \beta} \\
& -\eta_{\mu \alpha} k_{1 \beta} k_{2 \nu}-\eta_{\mu \beta} k_{1 \nu} k_{2 \alpha} \\
& +\eta_{\alpha \beta} k_{1 \mu} k_{2 \nu} .
\end{aligned}
$$

Since $m_{W}^{2}+k_{1} \cdot k_{2}=\left(\frac{m}{2}\right)^{2}+\left(\frac{m}{2}\right)^{2}=\frac{m^{2}}{2}$, we may write $W_{\mu \nu \alpha \beta}^{(W)}$ above as

$$
\begin{aligned}
W_{\mu \nu \alpha \beta}^{(W)}= & \frac{1}{2} \eta_{\mu \nu}\left(-\frac{m^{2}}{2} \eta_{\alpha \beta}+k_{1 \beta} k_{2 \alpha}\right) \\
& +\frac{m^{2}}{2} \eta_{\mu \alpha} \eta_{\nu \beta} \\
& -\eta_{\mu \alpha} k_{1 \beta} k_{2 \nu}-\eta_{\mu \beta} k_{1 \nu} k_{2 \alpha} \\
& +\eta_{\alpha \beta} k_{1 \mu} k_{2 \nu} .
\end{aligned}
$$


When we work with the simplified kinematical case (4.11), only the second line (4.18) of the above expression for $W_{\mu \nu}^{(W)}$ c contributes in the present calculation.

We calculate the angular distributions of the $\ell^{-}$and $\ell^{+}$for each of the possible initial gluon polarization states. We work in the $X_{2}$ rest frame, take the beam direction as the $z$-axis and write the gluon momenta as

$$
k_{1}^{\mu}=\left(k_{1}^{0}, k_{1}^{1}, k_{1}^{2}, k_{1}^{3}\right)=(k, 0,0, k), \quad k_{2}^{\mu}=\left(k_{2}^{0}, k_{2}^{1}, k_{2}^{2}, k_{2}^{3}\right)=(k, 0,0,-k) .
$$

The polarization vectors of the initial gluons are given by (2.2) and (2.3), and we denote the polarization of the gluon which has the momentum $k_{1}\left(k_{2}\right)$ in $(4.21)$ by $\epsilon_{1}^{g}\left(\epsilon_{2}^{g}\right)$.

\subsection{Angular correlations in $g g \rightarrow X_{2} \rightarrow W^{-} W^{+} \rightarrow \ell^{-} \ell^{+} \bar{\nu} \nu$}

We consider the decays $X_{2} \rightarrow W^{-} W^{+} \rightarrow \ell^{-} \ell^{+} \bar{\nu} \nu$ following $X_{2}$ production by $g g$ collisions with polarizations $\epsilon_{1}^{g}=\epsilon^{ \pm}, \epsilon_{2}^{g}=\epsilon^{ \pm}$. Collisions with $\epsilon_{1}^{g}=\epsilon^{+}, \epsilon_{2}^{g}=\epsilon^{+}$produce the $X_{2}$ in a $\left|J J^{z}\right\rangle=|2+2\rangle$ state, whereas collisions with $\epsilon_{1}^{g}=\epsilon^{-}, \epsilon_{2}^{g}=\epsilon^{-}$produce the $X_{2}$ in a $\left|J J^{z}\right\rangle=$ $|2-2\rangle$ state. As we saw in section 3.2, collisions with $\epsilon_{1}^{g}=\epsilon^{+}, \epsilon_{2}^{g}=\epsilon^{-}$and $\epsilon_{1}^{g}=\epsilon^{-}, \epsilon_{2}^{g}=\epsilon^{+}$ have vanishing amplitudes for producing the polarization state $\left|J J^{z}\right\rangle=|20\rangle$ of the $X_{2}$.

\subsection{1 $\left|J J^{z}\right\rangle=|2+2\rangle$}

When the polarizations of the initial gluons are given by $\epsilon_{1}^{g}=\epsilon_{2}^{g}=\epsilon^{+}$and the initial two-gluon polarization state is $|2+2\rangle$, the polarizations of $W^{-}$and $W^{+}$are also given by $\epsilon_{1}=\epsilon^{+}$and $\epsilon_{1}=\epsilon^{+}$. The amplitude

$$
\mathcal{M}=\mathcal{M}_{1} \mathcal{M}_{2}=\left(\bar{u}\left(p_{\ell^{-}}\right) \gamma^{\mu} \epsilon_{1 \mu}\left(1-\gamma_{5}\right) v\left(p_{\bar{\nu}}\right)\right)\left(\bar{u}\left(p_{\nu}\right) \gamma^{\mu} \epsilon_{2 \mu}\left(1-\gamma_{5}\right) v\left(p_{\ell^{+}}\right)\right),
$$

is then, from (4.3) and (4.8), given by:

$$
\frac{\mathcal{M}}{(2 \sqrt{2} p)^{2}}=-\left(1-\cos \theta_{1}\right)\left(1+\cos \theta_{2}\right) e^{i\left(\phi_{1}+\phi_{2}\right)},
$$

whose absolute square is independent of the azimuthal angles $\phi_{1,2}$ and proportional to:

$$
\left(1-\cos \theta_{1}\right)^{2}\left(1+\cos \theta_{2}\right)^{2} .
$$

In figure 4(a) we plot the quantity (4.24) multiplied by $\sin \theta_{1} \sin \theta_{2}$, to which $d^{2} \sigma / d \theta_{1} d \theta_{2}$ is proportional.

\subsection{2 $\left|J J^{z}\right\rangle=|2-2\rangle$}

Similarly, when the polarizations of the initial gluons are given by $\epsilon_{1}^{g}=\epsilon_{2}^{g}=\epsilon^{-}$and the initial two-gluon polarization state is $|2-2\rangle$, the polarizations of $W^{-}$and $W^{+}$are also given by $\epsilon_{1}=\epsilon^{-}$and $\epsilon_{1}=\epsilon^{-}$, and from (4.4) and (4.9) the final-state lepton-antilepton angular distribution is again independent of the azimuthal angles $\phi_{1,2}$ and proportional to

$$
\left(1+\cos \theta_{1}\right)^{2}\left(1-\cos \theta_{2}\right)^{2} .
$$

In figure $4(\mathrm{~b})$ we plot the quantity (4.25) times $\sin \theta_{1} \sin \theta_{2}$, to which $d^{2} \sigma / d \theta_{1} d \theta_{2}$ is proportional in this case. The sum of figures $4(\mathrm{a})$ and (b) is plotted in figure 4(c). 


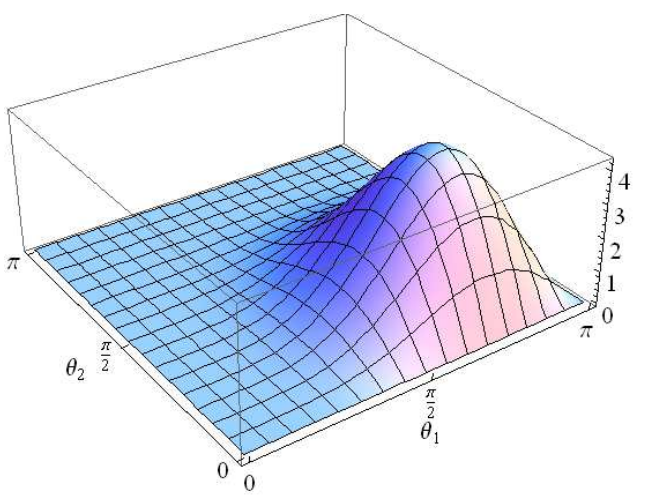

(a1)

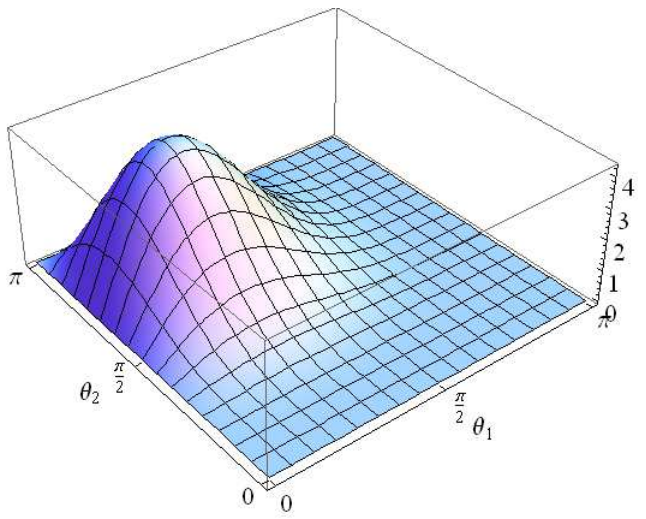

(b1)
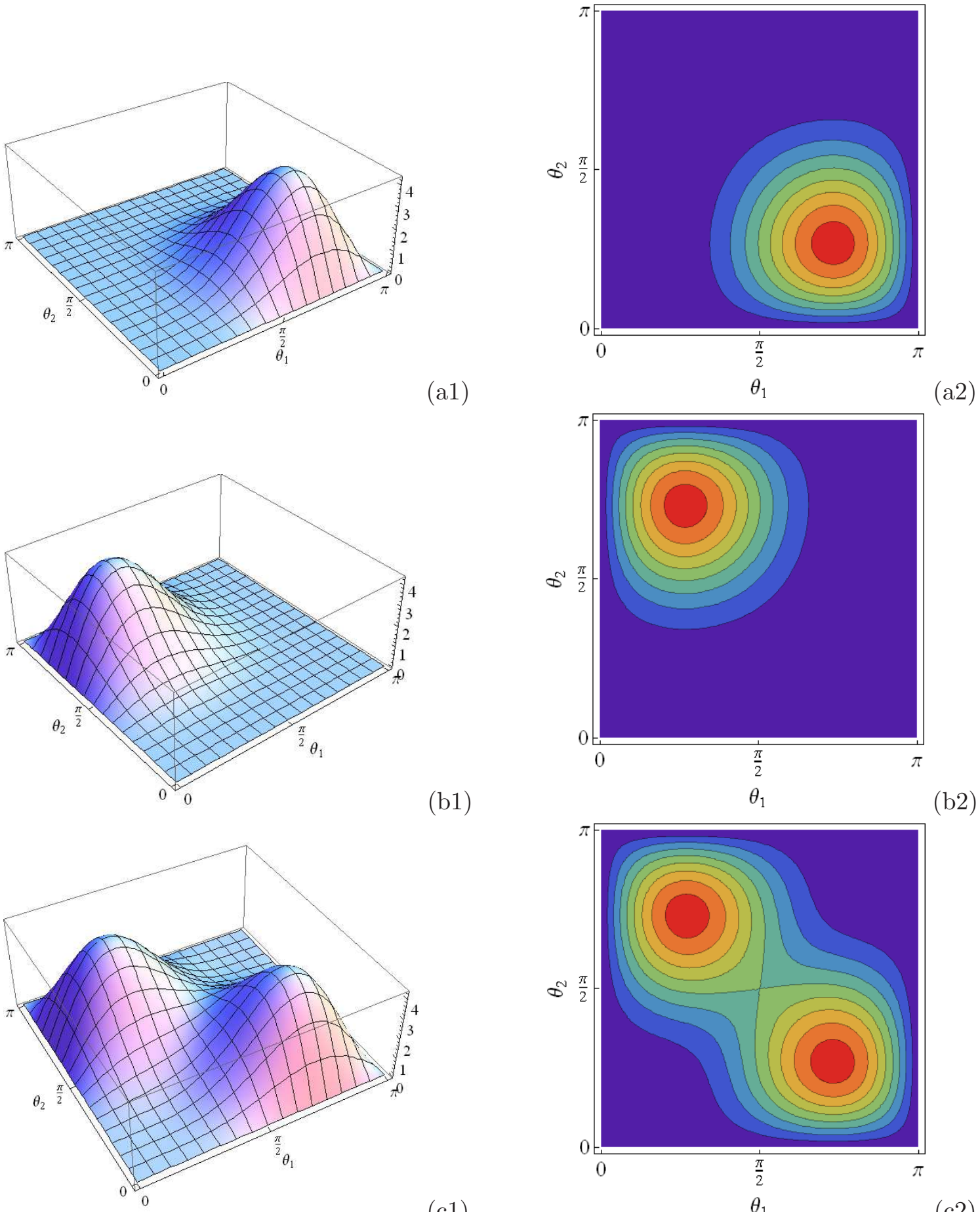

(b2)

(c1)

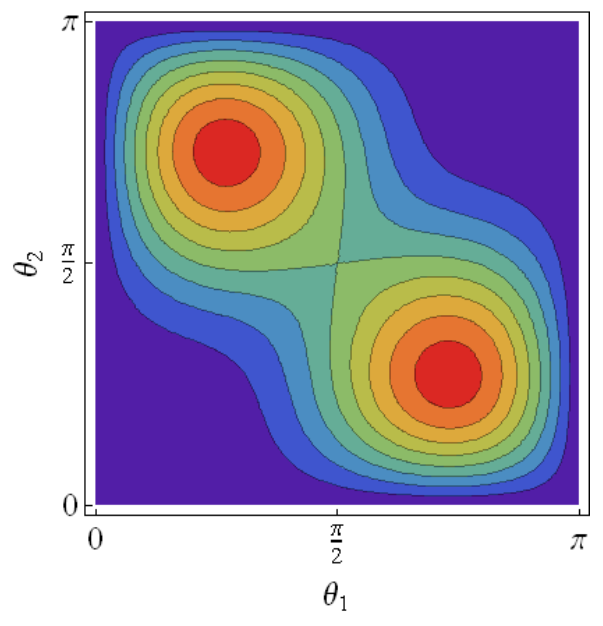

Figure 4. The angular distributions given by (a) (4.24) $\times \sin \theta_{1} \sin \theta_{2}$ for decays of the $\left|J J^{z}\right\rangle=$ $|2+2\rangle$ state of $X_{2} \rightarrow W^{-} W^{+} \rightarrow \ell^{-} \ell^{+} \bar{\nu} \nu$, (b) (4.25) $\times \sin \theta_{1} \sin \theta_{2}$ for decays of the $\left|J J^{z}\right\rangle=|2-2\rangle$ state of $X_{2} \rightarrow W^{-} W^{+} \rightarrow \ell^{-} \ell^{+} \bar{\nu} \nu$, and (c) the sum of (a) and (b). 


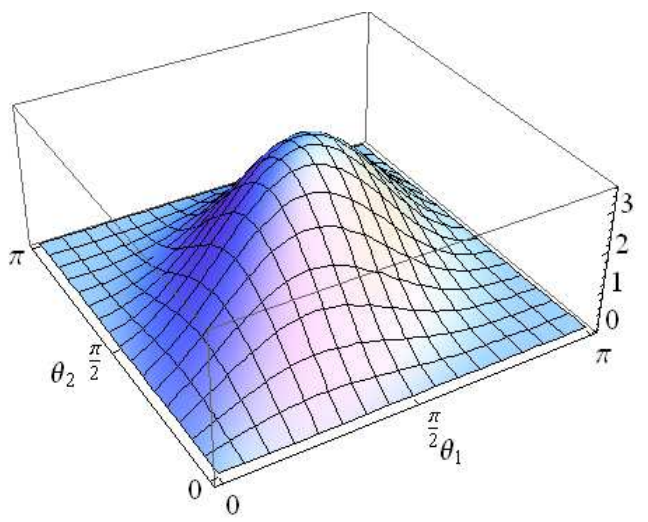

(a1)
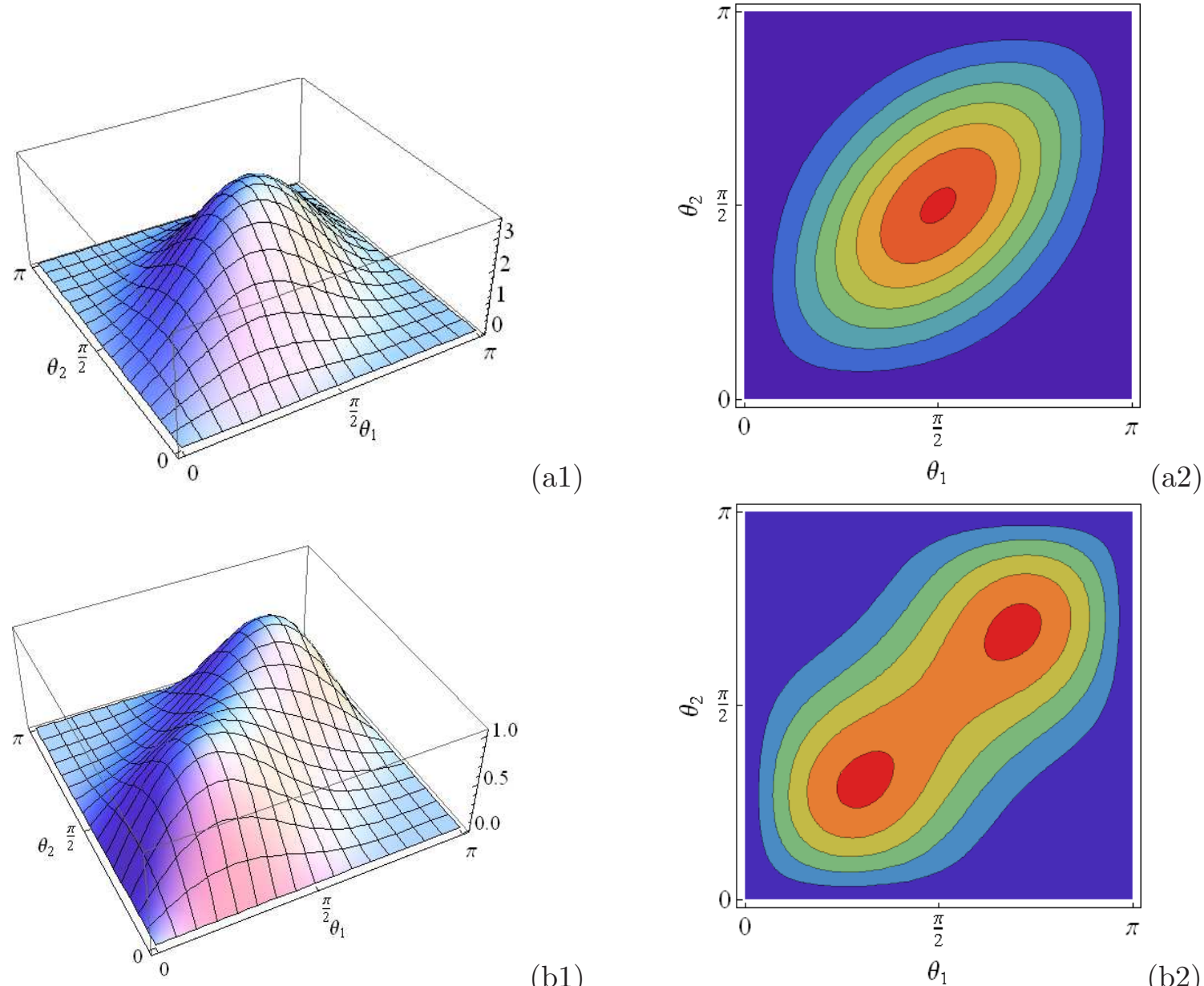

(b1)
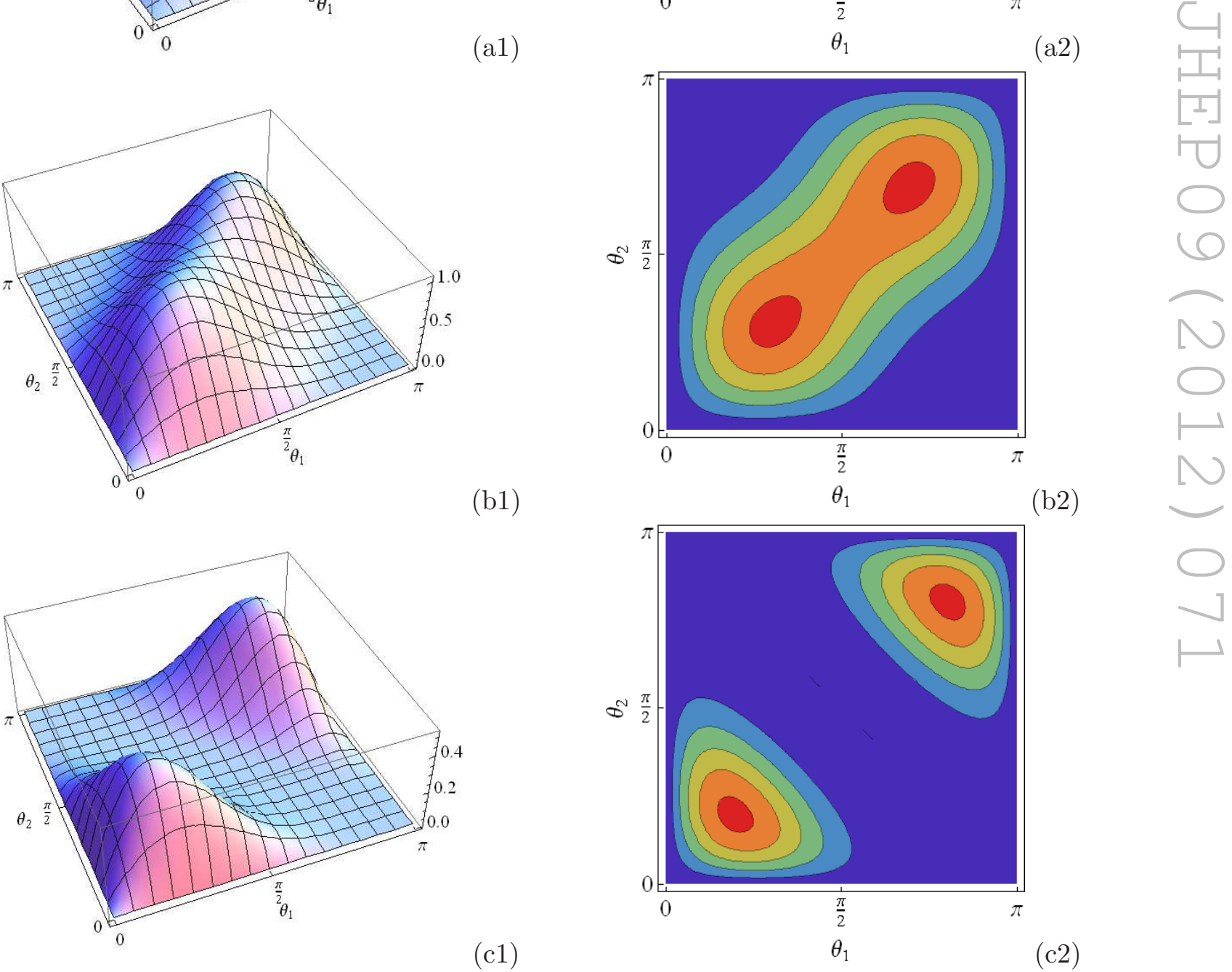

$(\mathrm{c} 1)$

Figure 5. The angular distributions for decays of $X_{0} \rightarrow W^{-} W^{+} \rightarrow \ell^{-} \ell^{+} \bar{\nu} \nu$ given by (4.32) $\times \sin \theta_{1} \sin \theta_{2}$ for (a) $\phi=0$, (b) $\pi / 2$ or $3 \pi / 2$, and (c) $\phi=\pi$. 


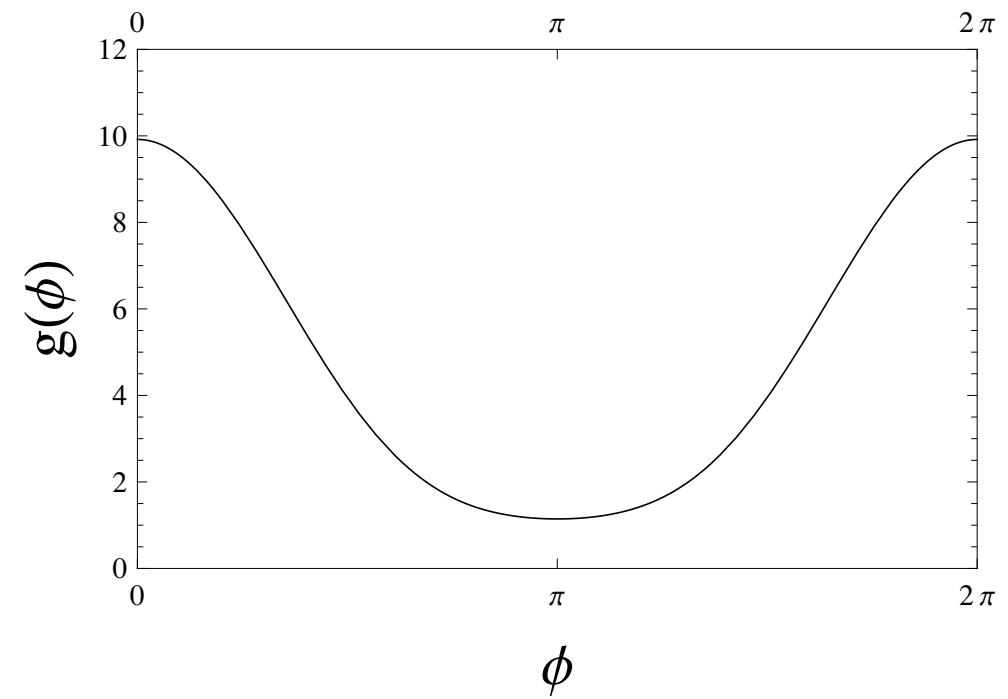

Figure 6. The azimuthal angular distribution $g(\phi)$ given in (4.33) for $X_{0} \rightarrow W^{-} W^{+} \rightarrow \ell^{-} \ell^{+} \bar{\nu} \nu$ decay.

\subsection{Angular correlations in $g g \rightarrow X_{0} \rightarrow W^{-} W^{+} \rightarrow \ell^{-} \ell^{+} \bar{\nu} \nu$}

For comparison, we now review the case of a spin-zero boson $X_{0}$. From the Clebsch-Gordan coefficients in

$$
|00\rangle=\sqrt{\frac{1}{3}}|1+1\rangle|1-1\rangle-\sqrt{\frac{1}{3}}|10\rangle|10\rangle+\sqrt{\frac{1}{3}}|1-1\rangle|1+1\rangle,
$$

at the vertex $X_{0} \rightarrow W^{-} W^{+}$for the $\left|J J^{z}\right\rangle=|00\rangle$ state of $X_{0}$, we see that the polarizations of the $W^{-}$and $W^{+}$are in the following coherent state:

$$
\sqrt{\frac{1}{3}} \epsilon_{1}^{+} \epsilon_{2}^{-}-\sqrt{\frac{1}{3}} \epsilon_{1}^{0} \epsilon_{2}^{0}+\sqrt{\frac{1}{3}} \epsilon_{1}^{-} \epsilon_{2}^{+} .
$$

The amplitude for the $W^{-} W^{+}$pair produced at the $X_{0} \rightarrow W^{-} W^{+}$vertex of the spin-zero Higgs particle given by (4.27) is proportional to $\eta_{\alpha \beta}$.

Then, from $(4.3),(4.4),(4.5),(4.8),(4.9),(4.10)$ and (4.27), we see that the amplitude $\mathcal{M}$ of (4.22) is given by the following coherent amplitude (omitting a factor $1 /(2 \sqrt{2} p)^{2}$ ):

$$
a e^{+i \phi}+b e^{-i \phi}+c,
$$

where $\phi \equiv \phi_{1}-\phi_{2}$ and

$$
\begin{aligned}
a & =-\sqrt{\frac{1}{3}}\left(1-\cos \theta_{1}\right)\left(1-\cos \theta_{2}\right) \\
b & =-\sqrt{\frac{1}{3}}\left(1+\cos \theta_{1}\right)\left(1+\cos \theta_{2}\right) \\
c & =-\sqrt{\frac{1}{3}} 2 \sin \theta_{1} \sin \theta_{2} .
\end{aligned}
$$


The absolute square of (4.28) is given by

$$
\left(a e^{-i \phi}+b e^{i \phi}+c\right)\left(a e^{i \phi}+b e^{-i \phi}+c\right)=a^{2}+b^{2}+c^{2}+2(a+b) c \cos \phi+2 a b \cos 2 \phi .
$$

In figure 5 we plot $(4.32) \times \sin \theta_{1} \sin \theta_{2}$ (which is proportional to $d^{2} \sigma / d \theta_{1} d \theta_{2}$ ) for $\phi=0$, $\pi / 2$ or $3 \pi / 2$, and $\pi$. The azimuthal angle distribution resulting from the integration

$$
g(\phi) \equiv \int_{0}^{\pi} \sin \theta_{1} d \theta_{1} \int_{0}^{\pi} \sin \theta_{2} d \theta_{2}[\text { eq. (4.32) }]
$$

is presented in figure 6 .

Comparing the results presented in figure 6 for the $X_{0}$ case with the fact that $g(\phi)$ is constant for the $X_{2}$ case as shown in the previous subsection, we see their clear difference in the angular correlations between the $\ell^{ \pm}$. This suggests that a careful study of the $\ell^{ \pm}$ angular distributions might offer some discrimination between the spin-two and spin-zero hypotheses. We note that the ATLAS [15-17] and CMS [18-25] $W^{+} W^{-}$event selections are based on the $\ell^{ \pm}$angular distributions predicted in the spin-zero case [31], see the angular distributions for the data, backgrounds and a possible $H \rightarrow W^{-} W^{+} \rightarrow \ell^{-} \ell^{+} \bar{\nu} \nu$ signal in $[32,33]$, and are likely to have reduced efficiencies for the spin-two case. However, any conclusions on the possible hypotheses would require realistic simulations of the $W^{-} W^{+} \rightarrow$ $\ell^{-} \ell^{+} \bar{\nu} \nu$ final states in an LHC detector.

\subsection{Dilepton invariant mass distributions}

We conclude this section by displaying in figure 7 the distributions in the $\ell^{-} \ell^{+}$invariant mass, $m_{l l}$, for $X_{0} \rightarrow W^{-} W^{+} \rightarrow \ell^{-} \ell^{+} \bar{\nu} \nu$ (a) and $X_{2} \rightarrow W^{-} W^{+} \rightarrow \ell^{-} \ell^{+} \bar{\nu} \nu$ (b). As could be expected from the differences in the angular distributions discussed above, and on the basis of helicity arguments, the $\ell^{-} \ell^{+}$invariant mass distribution peaks at a larger value in the $X_{2}$ case than in the $X_{0}$ case. This offers, in principle, another way to discriminate between the two possible spin assignments. We note that $[32,33]$ also compare data for $m_{l l}$ with simulations of $X_{0} \rightarrow W^{-} W^{+} \rightarrow \ell^{-} \ell^{+} \bar{\nu} \nu$ and experimental backgrounds.

\section{Summary}

We have presented in this paper analyses of the angular distributions that could be expected in the $\gamma \gamma$ and $W^{-} W^{+}$decays of a hypothetical spin-two state $X_{2}$ produced at the LHC via gluon-gluon collisions, assuming that its couplings coincide with those expected for a massive Kaluza-Klein graviton. Under this hypothesis, such a spin-two particle would be produced in a definite combination of polarization states, and the polar angle distribution of the $\gamma \gamma$ final state would be predictable and non-isotropic in the $X_{2}$ rest frame, and hence distinguishable in principle from the isotropic $\gamma \gamma$ decays of a hypothetical spin-zero boson $X_{0}$. Likewise, the angular correlations between the $\ell^{ \pm}$produced in $X_{2} \rightarrow W^{-} W^{+}$ decays are predictable and distinct from those in $X_{0} \rightarrow W^{-} W^{+}$decays. In this paper we have analyzed the case where the $W^{-} W^{+}$pair is at rest, which may be a suitable first approximation to the case of a state with mass $\sim 125 \mathrm{GeV}$ decaying into $W W^{*}$. 


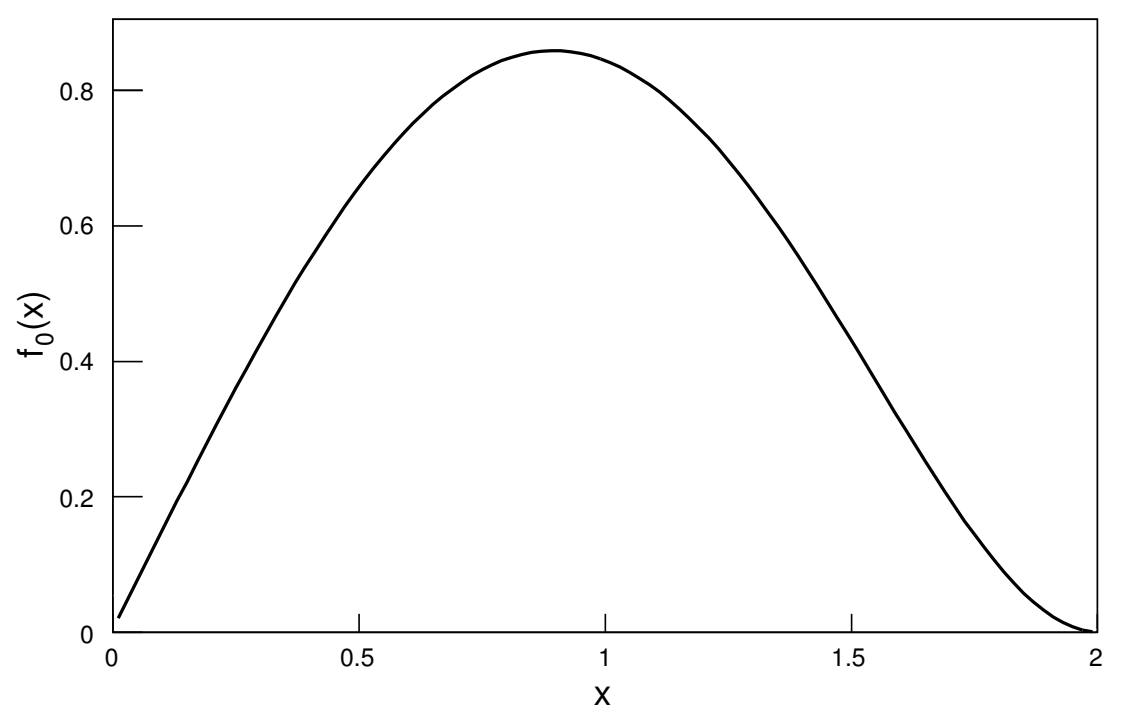

(a)

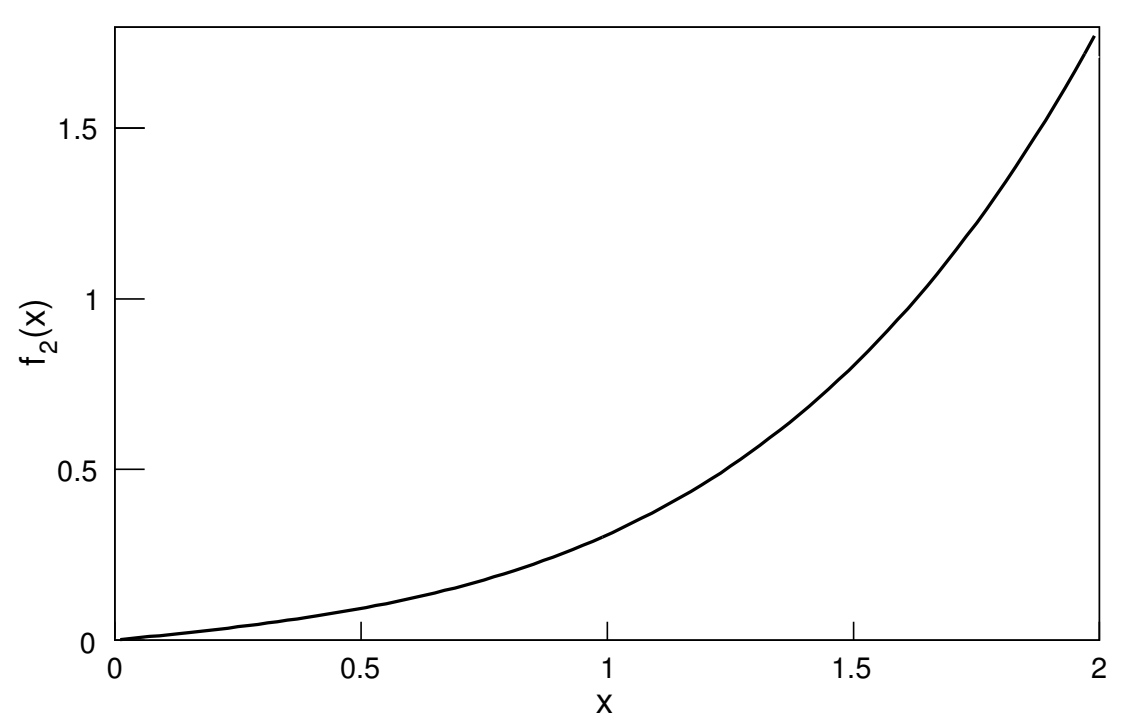

(b)

Figure 7. Distributions in the $\ell^{-} \ell^{+}$invariant mass, $m_{l l}$, for (a) the spin-zero case $X_{0}$ and (b) for the spin-two case $X_{2}$. The plotted quantities are $f_{0,2}(x) \equiv \frac{1}{\sigma} \frac{d \sigma}{d x}$ where $x \equiv 2 m_{l l} / m_{W}$, so that $x=2$ corresponds to $m_{l l}=m_{W}=\frac{m}{2}$, where $m$ is the mass of $X_{0}$ or $X_{2}$.

This analytical study would require detailed simulations for either ATLAS and/or CMS before one could conclude whether, in practice, these angular distributions could be used to provide supplementary information about the spin of the hypothetical particle that may be responsible for the excesses of events seen at $\sim 125 \mathrm{GeV}$ by both ATLAS and CMS. We think that the effort of making such simulations should be worthwhile, in view of the excesses of $\gamma \gamma$ and $W^{-} W^{+}$events already seen. 
As a preliminary step in this direction, we have initiated a project to simulate off-shell effects and the CMS and ATLAS experimental event selections, detection efficiencies and acceptances using PYTHIA and Delphes. Preliminary results of these simulations indicate that angular distributions discussed here do not vary substantially for candidate Higgs masses between 165 and $125 \mathrm{GeV}$, and continue to offer good discrimination between the spin-zero and spin-two hypotheses. Fuller details will be published elsewhere [34].

Many other approaches to analyzing the possible spin of a Higgs candidate rely on lepton angular correlations in $Z Z \rightarrow 4 \ell^{ \pm}$final states [2, 4, 7, 9-14]. These would provide considerably more information, but are limited by statistics likely to be available in the foreseeable future.

\section{Acknowledgments}

The work of J.E. is supported partly by the London Centre for Terauniverse Studies (LCTS), using funding from the European Research Council via the Advanced Investigator Grant 267352. The work of D.S.H. is supported partly by Korea Foundation for International Cooperation of Science \& Technology (KICOS) and National Research Foundation of Korea (2011-0005226). D.S.H. thanks CERN for its hospitality while working on this subject.

Open Access. This article is distributed under the terms of the Creative Commons Attribution License which permits any use, distribution and reproduction in any medium, provided the original author(s) and source are credited.

\section{References}

[1] D.J. Miller, S.Y. Choi, B. Eberle, M.M. Muhlleitner and P.M. Zerwas, Measuring the spin of the Higgs boson, Phys. Lett. B 505 (2001) 149 [hep-ph/0102023] [INSPIRE].

[2] S.Y. Choi, D.J. Miller, M.M. Muhlleitner and P.M. Zerwas, Identifying the Higgs spin and parity in decays to $Z$ pairs, Phys. Lett. B 553 (2003) 61 [hep-ph/0210077] [INSPIRE].

[3] K. Odagiri, On azimuthal spin correlations in Higgs plus jet events at LHC, JHEP 03 (2003) 009 [hep-ph/0212215] [INSPIRE].

[4] C.P. Buszello, I. Fleck, P. Marquard and J.J. van der Bij, Prospective analysis of spin- and $C P$-sensitive variables in $H \rightarrow Z Z \rightarrow l(1)+l(1)-l(2)+l(2)$ - at the $L H C$, Eur. Phys. J. C 32 (2004) 209 [hep-ph/0212396] [INSPIRE].

[5] A. Djouadi, The Anatomy of electro-weak symmetry breaking. I: The Higgs boson in the standard model, Phys. Rept. 457 (2008) 1 [hep-ph/0503172] [INSPIRE].

[6] C.P. Buszello and P. Marquard, Determination of spin and CP of the Higgs boson from $W B F$, hep-ph/0603209 [INSPIRE].

[7] A. Bredenstein, A. Denner, S. Dittmaier and M. Weber, Precise predictions for the Higgs-boson decay $H \rightarrow W W / Z Z \rightarrow 4$ leptons, Phys. Rev. D 74 (2006) 013004 [hep-ph/0604011] [INSPIRE]. 
[8] P.S. Bhupal Dev, A. Djouadi, R.M. Godbole, M.M. Muhlleitner and S.D. Rindani, Determining the CP properties of the Higgs boson, Phys. Rev. Lett. 100 (2008) 051801 [arXiv:0707.2878] [INSPIRE].

[9] R.M. Godbole, D.J.. Miller, and M.M. Muhlleitner, Aspects of CP-violation in the $H Z Z$ coupling at the LHC, JHEP 12 (2007) 031 [arXiv:0708.0458] [INSPIRE].

[10] K. Hagiwara, Q. Li and K. Mawatari, Jet angular correlation in vector-boson fusion processes at hadron colliders, JHEP 07 (2009) 101 [arXiv:0905.4314] [INSPIRE].

[11] Y. Gao et al., Spin determination of single-produced resonances at hadron colliders, Phys. Rev. D 81 (2010) 075022 [arXiv:1001.3396] [INSPIRE].

[12] A. De Rujula, J. Lykken, M. Pierini, C. Rogan and M. Spiropulu, Higgs look-alikes at the LHC, Phys. Rev. D 82 (2010) 013003 [arXiv:1001.5300] [inSPIRE].

[13] C. Englert, C. Hackstein and M. Spannowsky, Measuring spin and CP from semi-hadronic ZZ decays using jet substructure, Phys. Rev. D 82 (2010) 114024 [arXiv:1010.0676] [InSPIRE].

[14] U. De Sanctis, M. Fabbrichesi and A. Tonero, Telling the spin of the 'Higgs boson' at the LHC, Phys. Rev. D 84 (2011) 015013 [arXiv:1103.1973] [InSPIRE].

[15] ATLAS collaboration, G. Aad et al., Combined search for the Standard Model Higgs boson using up to $4.9 \mathrm{fb}-1$ of pp collision data at sqrt(s) $=7 \mathrm{TeV}$ with the ATLAS detector at the LHC, Phys. Lett. B 710 (2012) 49 [arXiv:1202.1408] [INSPIRE].

[16] ATLAS collaboration, G. Aad et al., Search for the Standard Model Higgs boson in the diphoton decay channel with $4.9 \mathrm{fb}-1$ of pp collisions at $\sqrt{s}=7$ TeV with ATLAS, Phys. Rev. Lett. 108 (2012) 111803 [arXiv:1202.1414] [INSPIRE].

[17] ATLAS collaboration, G. Aad et al., Search for the Standard Model Higgs boson in the decay channel $H \rightarrow Z Z\left(^{*}\right) \rightarrow 4 l$ with $4.8 \mathrm{fb}-1$ of pp collision data at $\sqrt{s}=7$ TeV with ATLAS, Phys. Lett. B 710 (2012) 383 [arXiv:1202.1415] [INSPIRE].

[18] CMS collaboration, S. Chatrchyan et al., Combined results of searches for the standard model Higgs boson in pp collisions at $\sqrt{s}=7$ TeV, Phys. Lett. B $\mathbf{7 1 0}$ (2012) 26 [arXiv: 1202.1488] [INSPIRE].

[19] CMS collaboration, S. Chatrchyan et al., Search for the standard model Higgs boson decaying into two photons in pp collisions at $\sqrt{s}=7$ TeV, Phys. Lett. B 710 (2012) 403 [arXiv: 1202.1487] [INSPIRE].

[20] CMS collaboration, S. Chatrchyan et al., Search for the standard model Higgs boson decaying to a $W$ pair in the fully leptonic final state in pp collisions at $\sqrt{s}=7 \mathrm{TeV}$,

Phys. Lett. B 710 (2012) 91 [arXiv:1202.1489] [INSPIRE].

[21] CMS collaboration, S. Chatrchyan et al., Search for the standard model Higgs boson in the decay channel $H$ to $Z Z$ to 4 leptons in pp collisions at $\sqrt{s}=7 \mathrm{TeV}$, Phys. Rev. Lett. 108 (2012) 111804 [arXiv:1202.1997] [INSPIRE].

[22] CMS collaboration, S. Chatrchyan et al., Search for the standard model Higgs boson in the H to ZZ to $2 \mathrm{l} 2 \mathrm{2nu}$ channel in pp collisions at $\sqrt{s}=7 \mathrm{TeV}$, JHEP 03 (2012) 040 [arXiv: 1202.3478] [INSPIRE].

[23] CMS collaboration, S. Chatrchyan et al., Search for the standard model Higgs boson in the H to ZZ to ll tau tau decay channel in pp collisions at $\sqrt{s}=7$ TeV, JHEP 03 (2012) 081 [arXiv: 1202.3617] [INSPIRE]. 
[24] CMS collaboration, S. Chatrchyan et al., Search for neutral Higgs bosons decaying to tau pairs in pp collisions at $\sqrt{s}=7$ TeV, Phys. Lett. B 713 (2012) 68 [arXiv:1202.4083] [INSPIRE].

[25] CMS collaboration, S. Chatrchyan et al., Search for the standard model Higgs boson decaying to bottom quarks in pp collisions at $\sqrt{s}=7$ TeV, Phys. Lett. B 710 (2012) 284 [arXiv:1202.4195] [INSPIRE].

[26] L.A. Anchordoqui, H. Goldberg and T.R. Taylor, Decay widths of lowest massive Regge excitations of open strings, Phys. Lett. B 668 (2008) 373 [arXiv:0806.3420] [INSPIRE].

[27] G.F. Giudice, R. Rattazzi and J.D. Wells, Quantum gravity and extra dimensions at high-energy colliders, Nucl. Phys. B 544 (1999) 3 [hep-ph/9811291] [INSPIRE].

[28] T. Han, J.D. Lykken and R.-J. Zhang, On Kaluza-Klein states from large extra dimensions, Phys. Rev. D 59 (1999) 105006 [hep-ph/9811350] [INSPIRE].

[29] T. Gleisberg et al., Helicity formalism for spin-2 particles, JHEP 09 (2003) 001 [hep-ph/0306182] [INSPIRE].

[30] R. Fok, C. Guimaraes, R. Lewis and V. Sanz, It is a Graviton! or maybe not,

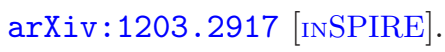

[31] M. Dittmar and H.K. Dreiner, How to find a Higgs boson with a mass between 155 GeV 180 GeV at the LHC, Phys. Rev. D 55 (1997) 167 [hep-ph/9608317] [InSPIRE].

[32] ATLAS collaboration, G. Aad et al., Observation of a new particle in the search for the Standard Model Higgs boson with the ATLAS detector at the LHC, arXiv:1207.7214.

[33] CMS collaboration, S. Chatrchyan et al., Observation of a new boson at a mass of 125 GeV with the CMS experiment at the LHC, arXiv:1207.7235.

[34] J.R. Ellis, R. Fok, D.S. Hwang, V. Sanz and T. You, Towards the Determination of the 'Higgs' Spin and Parity, in preparation. 